Bryn Mawr College

Scholarship, Research, and Creative Work at Bryn Mawr

College

1986

\title{
Deuteron Zeeman Relaxation of CD4 in the Isotropic Liquid, the Liquid Crystalline, and the Solid State of Several Substances
}

Peter A. Beckmann

Bryn Mawr College, pbeckman@brynmawr.edu

Myer Bloom

E. Elliott Burnell

Let us know how access to this document benefits you.

Follow this and additional works at: http://repository.brynmawr.edu/physics_pubs

Part of the Physics Commons

\section{Custom Citation}

P.A. Beckmann, M. Bloom and E.E. Burnell, J. Chem. Phys 84, 5898 (1986).

This paper is posted at Scholarship, Research, and Creative Work at Bryn Mawr College. http://repository.brynmawr.edu/physics_pubs/48 


\title{
Deuteron Zeeman relaxation of $\mathrm{CD}_{4}$ in the isotropic liquid, the liquid crystalline, and the solid state of several substances
}

\author{
Peter A. Beckmann ${ }^{\text {a) }}$ \\ Department of Chemistry, University of British Columbia, Vancouver, British Columbia, V6T 1W5, Canada \\ Myer Bloom \\ Department of Physics, University of British Columbia, Vancouver, British Columbia, V6T IW5, Canada \\ E. Elliott Burnell \\ Department of Chemistry, University of British Columbia, Vancouver, British Columbia, V6T 1W5, Canada
}

(Received 29 October 1985; accepted 11 February 1986)

\begin{abstract}
Measurement of deuteron Zeeman relaxation rates of $\mathrm{CD}_{4}$ dissolved in benzene, hexane, and the liquid crystals MBBA, EBBA, and Merck ZLI-1132 and in pure $\mathrm{CD}_{4}$ gas as a function of temperature at 30.7 and $61.4 \mathrm{MHz}$ shows that the $\mathrm{CD}_{4}$ is uniformly dispersed in the liquid solvents but resides in gas pockets when the solvents are in the solid state. Effects of centrifugal distortion were observed in the gas phase. The relaxation rate was found to be nearly independent of solvent, temperature, and pressure for the methane-liquid mixtures. This result is explained in terms of the extended diffusion model for the combined effects of free molecular rotation and collisions on the spectral density of quadrupolar interactions when the collisional and mean free rotational periods are of the same order of magnitude. It can also be interpreted in terms of the Fokker-Planck-Langevin model for rotational Brownian motion.
\end{abstract}

\section{INTRODUCTION}

Methane is an ideal molecule for studying fundamental molecular processes using nuclear magnetic resonance (NMR). The methane molecule is small and highly symmetric and a great deal is known about its electronic, vibrational, and rotational structure. NMR studies in pure $\mathrm{CH}_{\mathbf{4}}$ and its four deuterated analogs have yielded considerable information concerning fundamental intermolecular interactions and collisional processes under a variety of conditions, including the following three examples: (1) The NMR properties of solid methane at low temperatures are very sensitive to subtle effects associated with the permutation symmetry of the nuclear spins and the rotational tunneling of the molecules. ${ }^{1}(2)$ The small NMR splittings observed in methane and its deuterated analogs dissolved in nematic liquid crystals provide information on the general nature of the average molecular fields in anisotropic fluids. ${ }^{2}$ (3) Nuclear spin relaxation in methane gas provides information on collisional cross sections for molecular reorientation and on details of the spin-rotation, nuclear quadrupole, and the intramolecular nuclear dipole-dipole interactions. ${ }^{3-6}$

The present study began as an empirical study of nuclear (deuteron) spin relaxation of $\mathrm{CD}_{4}$ dissolved in various isotropic and anisotropic liquids in order to explore how the molecular reorientation rates of small molecules in solution are influenced by the orientational order of the solvent. To our surprise, the observed relaxation rates were all virtually identical and independent of temperature and pressure.

The main purpose of this paper is to present these experimental results and to explain the observed insensitivity of the relaxation rates in liquid solutions to the detailed molecular properties of the liquids. We are led by these results to focus our attention on the combined influence of free mo-

\footnotetext{
*) On leave, 1984-1985, from the Department of Physics, Bryn Mawr College, Bryn Mawr, Pennsylvania 19010.
}

lecular rotation and collision induced molecular reorientation on the correlation function of the intramolecular quadrupolar interactions which are responsible for the deuteron spin relaxation in $\mathrm{CD}_{4}$. Since the quadrupole coupling constant is known, it is possible to show from the universal value of the relaxation rate in all of the liquids studied that the collision frequency in all these liquids is, coincidentally, close to the average rotational frequency of $\mathrm{CD}_{4}$. Under these conditions, we shall show that the spectral density of the quadrupolar interactions responsible for deuteron spin relaxation in $\mathrm{CD}_{4}$ is only weakly dependent on the collision frequency thus accounting for the universal behavior observed in liquid solution.

In contrast to the results in liquid solutions, when the solvent is in the solid phase the $\mathrm{CD}_{4}$ relaxation does depend on pressure. In addition, the relaxation rates are equal to those measured for the pure gas at the same pressure and temperature. Although we shall refer to these solid samples as solid solutions, they are in fact not homogeneous.

\section{THE EXPERIMENT}

Samples were made by dissolving $\mathrm{CD}_{4}$ gas in benzene, hexane, $N$-p-methoxybenzylidene- $p^{\prime}-n$-butylaniline (MBBA), $N$ - $p$-ethoxybenzylidene- $p^{\prime}-n$-butylaniline (EBBA), and Merck ZLI 1132, a mixture of three phenylcylclohexanes and one biphenylcyclohexane (1132). The liquids were well degassed on a vacuum line with many freeze-pumpthaw cycles. The $\mathrm{CD}_{4}$ gas ( 99 at. \% D) was obtained from Merck, Sharp, and Dohme and was used without purification. Samples using MBBA, EBBA, and 1132 as solvents were made by condensing $18 \mathrm{~atm}$ of $\mathrm{CD}_{4}$ gas at room temperature above the solvent. Samples of benzene and hexane were made with $\mathrm{CD}_{4}$ pressures of $15 \mathrm{~atm}$ and one benzene sample was made with a $\mathrm{CD}_{4}$ pressure of $1.0 \mathrm{~atm}$. In all samples, the ratio of the volume of the $\mathrm{CD}_{4}$ gas to that of the 
liquid solvent or solid solvent plus dissolved $\mathrm{CD}_{4}$ was about four to one. In addition, four gas samples were made with pressures of $6,1,0.5$, and $0.1 \mathrm{~atm} \mathrm{CD}_{4}$ at room temperature. All pressures are accurate to $\pm 20 \%$.

The deuteron magnetic resonance spectra and the deuteron Zeeman relaxation rates were measured on three Bruker nuclear magnetic resonance spectrometers: a WH400 operating at $61.4 \mathrm{MHz}$, a CXP-200 operating at 30.7 $\mathrm{MHz}$, and a considerably modernized BKR-322s operating at $30.7 \mathrm{MHz}$. To accommodate the long sample tubes, saddle coil inserts were constructed for the high-power, broadband probes used with the high-power CXP and BKR spectrometers. A standard high-resolution probe was used with the relatively low-power WH-400 spectrometer. Temperature was varied and controlled using either nitrogen gas (initially at $77 \mathrm{~K}$ ) or air. Temperatures are known to $\pm 5 \mathrm{~K}$ for the benzene and hexane samples and to $\pm 1 \mathrm{~K}$ for the liquid crystalline samples where the observed quadrupolar splittings provide internal thermometry. The temperature gradient along the sample varied between 1 and $3 \mathrm{~K}$ depending on the size of the sample.

\section{RESULTS}

The NMR spectrum of $\mathrm{CD}_{4}$ dissolved in an isotropic liquid is a single line. When the solvent is in the nematic state the $\mathrm{CD}_{4}$ deuteron spectrum is dominated by a quadrupolar doublet. Spectra ${ }^{7}$ along with a thorough discussion concerning the details of the quadrupolar, dipolar, and scalar couplings observed in the spherical top $\mathrm{CD}_{4}$ in nematic phases $^{7-9}$ are presented elsewhere. The temperature dependence of the quadrupolar splittings for $\mathrm{CD}_{4}$ in EBBA is shown in Fig. 1. Typical spectra for $\mathrm{CD}_{4}$ in MBBA, as well as the temperature dependence of the quadrupolar splittings are also given elsewhere. ${ }^{10,11}$

When the solvent is in the solid state the spectrum of $\mathrm{CD}_{4}$ is a single line whose $\Delta v$ is determined by magnet inhomogeneity. This was poor at low temperatures, ranging from 10 to $100 \mathrm{~Hz}$. Hence it is not possible to determine a spin-spin relaxation rate from the linewidth. However, it is clear that $\Delta v$ is orders of magnitude less than a typical solid

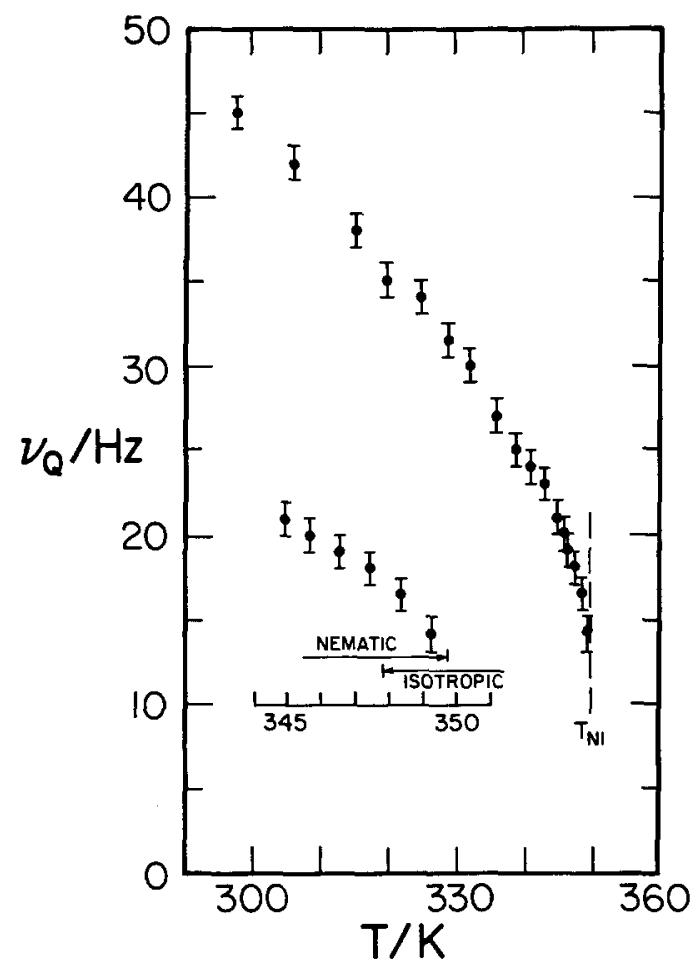

FIG. 1. Solute $\mathrm{CD}_{4}$ deuteron quadrupolar splitting $v_{Q}$ vs temperature $T$ for $\mathrm{CD}_{4}$ in EBBA. The inset shows the two-phase region, due predominantly to temperature inhomogeneity, on an expanded scale.

line and that $\mathrm{CD}_{4}$ is undergoing rapid fluid-like motion.

Deuteron Zeeman relaxation rates $R=T_{1}^{-1}$ of the solute $\mathrm{CD}_{4}$ were measured at 30.7 and $61.4 \mathrm{MHz}$ as a function of temperature $T$ in three states of the solvents: solid $S$, nematic $N$, and isotropic liquid $I$ as indicated in Table I. Relaxation measurements were also performed on the gas. $R$ was measured using the usual inversion recovery pulse sequence with a relaxation delay of $\geqslant 8 T_{1}$. Appropriate phase cycling was used to reduce systematic errors. The $R$ vs $T$ results for the $S, N$, and $I$ samples are presented in Figs. 2 and 3 . For the long $T_{1}$ values, the experiments took between 4 and $24 \mathrm{~h}$ depending on signal to noise. The error bars in the figures are liberal estimates.

TABLE I. Experimental and theoretical parameters.

\begin{tabular}{|c|c|c|c|c|c|c|c|c|}
\hline $\begin{array}{c}\text { Sample } \\
\text { No. }\end{array}$ & Solvent & $\begin{array}{c}\mathrm{CD}_{4} \\
P / \mathrm{atm}\end{array}$ & $\begin{array}{c}\text { Range of } \\
T / \mathrm{K}\end{array}$ & $\begin{array}{l}\text { Solvent } \\
\text { state }^{a}\end{array}$ & $\begin{array}{c}\text { Range of } \\
R / \mathrm{s}^{-1}\end{array}$ & $\begin{array}{c}\text { Range of } \\
\tau_{\theta} / \mathrm{ps}^{\mathrm{b}}\end{array}$ & $\begin{array}{c}\text { Range of } \\
\omega_{R}^{-1} / \mathrm{ps}\end{array}$ & $\begin{array}{c}\text { Range of } \\
\omega_{R} \tau_{\theta}^{b}\end{array}$ \\
\hline I & benzene & $15 \pm 3$ & $280-340$ & $I$ & $0.050-0.060$ & $0.10-0.13$ & $0.15-0.17$ & $0.59-0.87$ \\
\hline II & benzene & $1.0 \pm 0.2$ & $315-355$ & $I$ & $0.050-0.10$ & $0.10-0.21$ & $0.15-0.16$ & $0.63-1.4$ \\
\hline III & hexane & $15 \pm 3$ & $315-355$ & $I$ & $0.050-0.060$ & $0.10-0.13$ & $0.15-0.16$ & $0.63-0.87$ \\
\hline IV & MBBA & $18 \pm 4$ & $320-380$ & $I$ & $0.050-0.075$ & $0.10-0.16$ & $0.15-0.16$ & $0.63-1.1$ \\
\hline $\mathrm{V}$ & EBBA & $18 \pm 4$ & 360 & $I$ & $0.057-0.065$ & $0.12-0.14$ & 0.15 & $0.80-0.93$ \\
\hline VI & 1132 & $18 \pm 4$ & 360 & $I$ & $0.053-0.066$ & $0.11-0.14$ & 0.15 & $0.73-0.93$ \\
\hline IV & MBBA & $18 \pm 4$ & $280-340$ & $N$ & $0.060-0.095$ & $0.13-0.20$ & $0.15-0.17$ & $0.87-1.3$ \\
\hline V & EBBA & $18 \pm 4$ & $295-345$ & $N$ & $0.056-0.072$ & $0.12-0.15$ & $0.15-0.16$ & $0.80-0.94$ \\
\hline VI & 1132 & $18 \pm 4$ & $295-345$ & $N$ & $0.050-0.090$ & $0.10-0.25$ & $0.15-0.16$ & $0.63-1.7$ \\
\hline I & benzene & $15 \pm 3$ & $180-240$ & $S$ & $0.48-0.56$ & $1.0-1.2$ & $0.18-0.21$ & $4.8-6.7$ \\
\hline II & benzene & $1.0 \pm 0.2$ & $154-240$ & $S$ & $3.0-12$ & $6.3-25$ & $0.18-0.23$ & $27-139$ \\
\hline IV & MBBA & $18 \pm 4$ & $200-260$ & $S$ & $0.38-0.41$ & $0.79-0.85$ & $0.18-0.20$ & $4.0-4.7$ \\
\hline
\end{tabular}

a $I=$ isotropic liquid, $N=$ nematic, $S=$ solid.

${ }^{b}$ These numbers have been computed using the value $e V_{z z} Q / h=168 \mathrm{kHz}$. We believe that this value represents an approximate lower bound to the vibrationally averaged value of the quadrupole coupling constant. An upper bound is set by the equilibrium value of $189 \mathrm{kHz}$ (Refs. 2 and 9 ). This upper bound would give values of $\tau_{\theta}$ and $\omega_{R} \tau_{\theta}$ that are $21 \%$ smaller than those reported. 

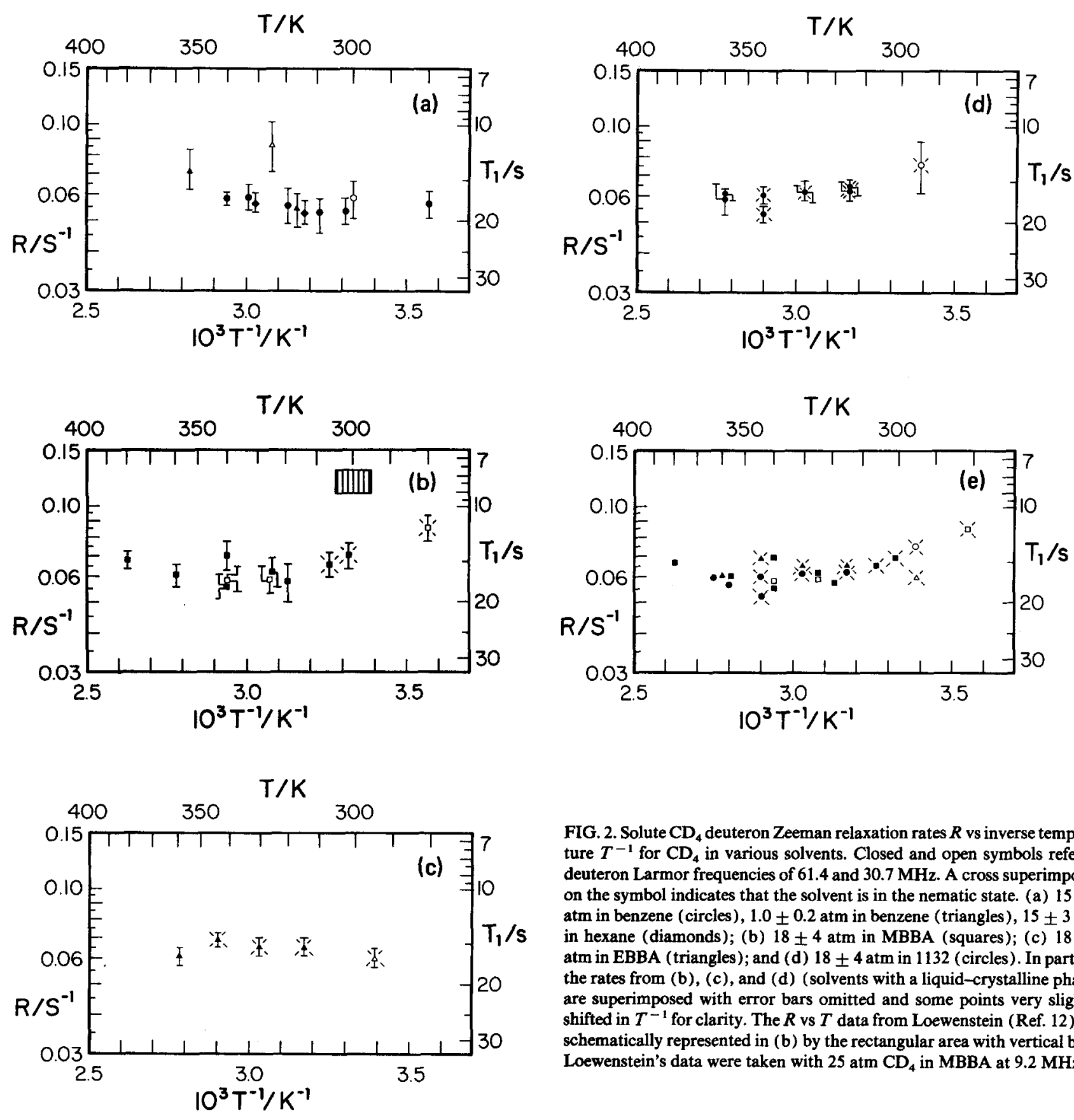

FIG. 2. Solute $\mathrm{CD}_{4}$ deuteron Zeeman relaxation rates $R$ vs inverse temperature $T^{-1}$ for $\mathrm{CD}_{4}$ in various solvents. Closed and open symbols refer to deuteron Larmor frequencies of 61.4 and $30.7 \mathrm{MHz}$. A cross superimposed on the symbol indicates that the solvent is in the nematic state. (a) $15 \pm 3$ atm in benzene (circles), $1.0 \pm 0.2 \mathrm{~atm}$ in benzene (triangles), $15 \pm 3 \mathrm{~atm}$ in hexane (diamonds); (b) $18 \pm 4 \mathrm{~atm}$ in MBBA (squares); (c) $18 \pm 4$ atm in EBBA (triangles); and (d) $18 \pm 4$ atm in 1132 (circles). In part (e) the rates from (b), (c), and (d) (solvents with a liquid-crystalline phase) are superimposed with error bars omitted and some points very slightly shifted in $T^{-1}$ for clarity. The $R$ vs $T$ data from Loewenstein (Ref. 12) are schematically represented in (b) by the rectangular area with vertical bars. Loewenstein's data were taken with $25 \mathrm{~atm} \mathrm{CD}_{4}$ in MBBA at $9.2 \mathrm{MHz}$.

The results in Figs. 2 and 3 are summarized in the sixth column of Table I. It is convenient to divide the results into two groups: solvent in a liquid state and solvent in the solid state. In the first nine rows of Table I where the solvent is in either the nematic $(N)$ or isotropic liquid $(I)$ state, $R$ is generally between 0.05 and $0.07 \mathrm{~s}^{-1}$. The benzene and hexane samples may have a slightly smaller $R$ than the MBBA, EBBA, and 1132 samples in the vicinity of $310 \mathrm{~K}$ but other than that there is little reason to distinguish between the different solvents and between the two Larmor frequencies. Of special note is that there is little or no temperature dependence in $R$. Also there is no difference between the 1 and 15 atm samples of benzene. The single high $R$ at $31 \mathrm{MHz}$ for the low-pressure benzene sample [Fig. 2(a)] is considered an experimental anomaly: $\mathrm{S} / \mathrm{N}$ was low and the experiment took $15 \mathrm{~h}$ on the CXP. Even though great care was taken to

rule out systematic errors it is impossible to consider all possible sources of such errors on commercial multifrequency spectrometers when $\mathrm{S} / \mathrm{N}$ is very low and $R$ very small (i.e., very long repetition periods). The second group in Table $I$ is the last three rows where the solvent is in the solid (S) state. The $R$ vs $T$ values are shown in Fig. 3. Here there is a marked dependence on $\mathrm{CD}_{4}$ pressure.

Loewenstein has measured $R$ vs $T$ at $9.2 \mathrm{MHz}$ in a sample with about $25 \mathrm{~atm}$ pressure of $\mathrm{CD}_{4}$ in $\mathrm{MBBA} .{ }^{12}$ The five values of $R$ when the MBBA is nematic are schematically represented by the rectangular region with vertical bars in Fig. 2(b). These $R$ values are more than twice the values found in the present 18 atm pressure sample. We do not understand the discrepancy between Loewenstein's and our results. However, effects such as paramagnetic impurities serve to increase $R$ and smaller $R$ values are normally con- 


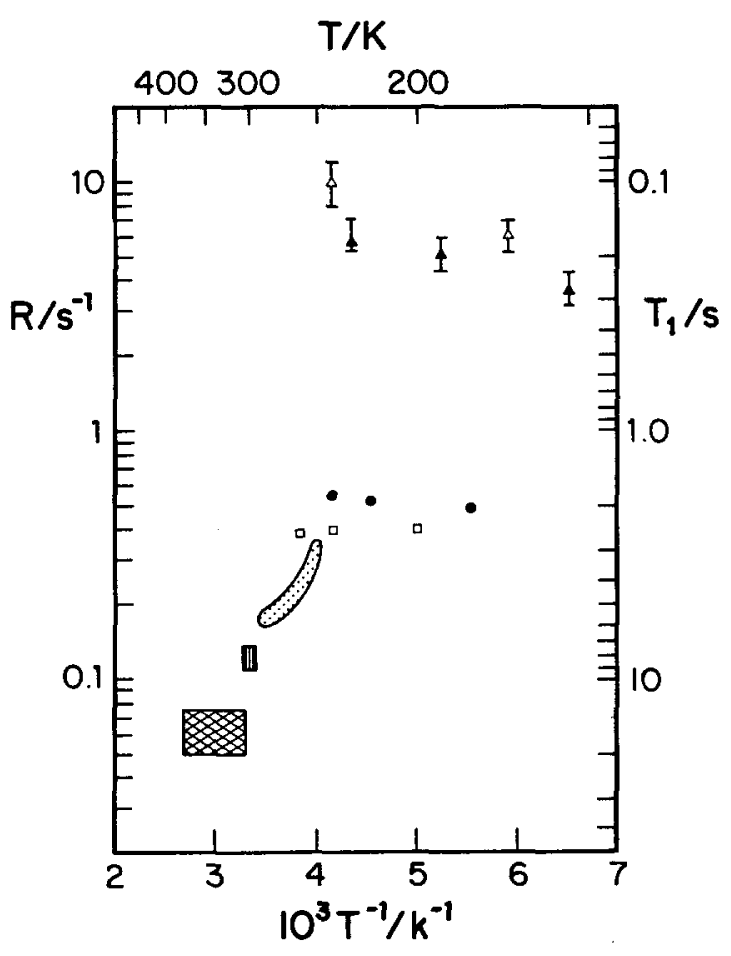

FIG. 3. Solute $\mathrm{CD}_{4}$ deuteron Zeeman relaxation rates $R$ vs inverse temperature $T^{-1}$ in the solid state of the solvents: $\mathrm{MBBA}$ with a $\mathrm{CD}_{4}$ room-temperature pressure of $P=18 \pm 4$ atm (squares); benzene with $P=15 \pm 3$ atm (circles); and benzene with $P=1.0 \pm 0.2 \mathrm{~atm}$ (triangles). Closed and open symbols refer to deuteron Larmor frequencies of 61.4 and $30.7 \mathrm{MHz}$. Where no error bars are shown, the uncertainties are within the size of the symbols. The $R$ vs $T^{-1}$ when the solvents are in the liquid state (Fig. 2) are shown schematically by the crosshatched region. Loewenstein's liquid values are shown by the rectangular area with vertical bars and his solid values by the dot filled region (Ref. 12).

sidered more reliable. Loewenstein's data are reproduced in Fig. 3. Also in Fig. 3 are Loewenstein's $R$ vs $T$ data when the solvent is in the solid state, represented by the dot-filled region.

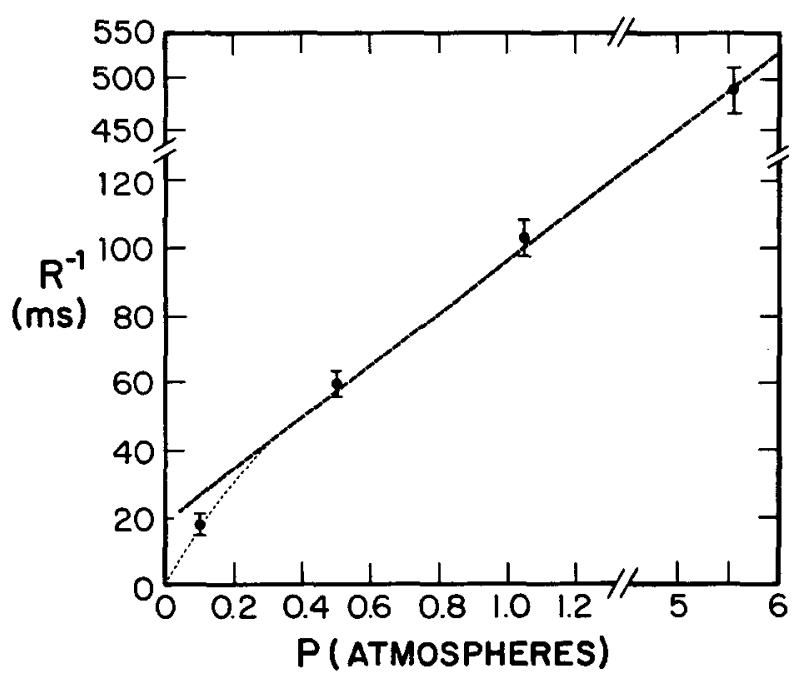

FIG. 4. Deuteron Zeeman relaxation time $R^{-1}$ vs pressure $P$ for $\mathrm{CD}_{4}$ gas at $T=295 \mathrm{~K}$. The dashed line is the fit to Eq. (12) and the dotted line is drawn to guide the eye.
Relaxation measurements have also been performed at $295 \mathrm{~K}$ on gaseous $\mathrm{CD}_{4}$ at four densities. The results are presented in Fig. 4.

\section{THEORETICAL CONSIDERATIONS}

The deuteron relaxation rate for isotropically reorienting $\mathrm{CD}_{4}$ molecules in the short correlation time regime can be expressed in the following simple form ${ }^{13}$ :

$$
1 / T_{1}=1 / T_{2}=R=\left(3 \pi^{2} / 2\right)\left(e V_{z z} Q / h\right)^{2} j(0),
$$

where $e V_{z z} Q / h$ is the quadrupolar coupling constant of the deuterons in $\mathrm{CD}_{4}$. Recent experiments on deuterated methanes dissolved in nematic solvents give a value of $189 \pm 3$ $\mathrm{kHz}$ for $\left(e V_{z z} Q / h\right)^{\mathrm{eq}}$, the quadrupolar coupling constant for the equilibrium geometry for $\mathrm{CD}_{4}{ }^{2,9}$ The value averaged over molecular vibrations will be somewhat lower. In this paper we shall use the commonly accepted experimental value for hydrocarbons of $168 \mathrm{kHz}^{14}$ Early NMR relaxation experiments of gaseous $\mathrm{CD}_{4}$ indicated a value between 130 and $200 \mathrm{kHz}{ }^{6}$ The quantity $j(0)$ is the reduced spectral density of the quadrupolar interactions at zero frequency, i.e., $\tau_{\theta} \equiv j(0)$ has the dimension of time and is the area under the reduced $[g(0)=1]$ correlation function of $Y_{2 m}[\Omega(t)]$ associated with the orientation $\Omega(t)$ of a particular CD bond in a $\mathrm{CD}_{4}$ molecule:

$$
\tau_{\theta} \equiv j(0)=\int_{0}^{\infty} g(t) d t .
$$

In liquid-crystal solvents, methane exhibits anisotropic couplings ${ }^{7}$ and there is a small correction term in Eq. (1). ${ }^{15,16}$ This term can be ignored since its effect is to change Eq. (1) by a fractional amount of order $S_{\mathrm{CD}}$, where $S_{\mathrm{CD}}$ $\left(\sim 10^{-4}\right.$ for $\left.\mathrm{CD}_{4}\right)$ is the order parameter for a $\mathrm{CD}$ bond.

The time dependence of $g(t)$ is influenced both by free rotation and by collision induced reorientation of the $\mathrm{CD}_{4}$ molecules. The short correlation time regime in a Zeeman relaxation study corresponds to $\omega_{0} \tau_{\theta}<1$, where $\omega_{0}$ is the deuteron Larmor angular frequency. We shall now consider three models which relate $\tau_{\theta}$ to dynamical molecular processes.

Model A. Assumption that the processes of free molecular rotation and molecular reorientation due to collisions are independent of each other. In this "strong collision model,"

$$
g(t)=[g(t)]_{R}[g(t)]_{C},
$$

where the subscripts $R$ and $C$ refer to free rotation and collisions. In this model it is assumed that the mean time $\tau_{C}$ between collisions which randomize the molecular orientation is given by

$$
\tau_{C}=\int_{0}^{\infty}[g(t)]_{C} d t
$$

The rotation is governed by the moment of inertia, $I_{0}=1.1 \times 10^{-46} \mathrm{~kg} \mathrm{~m}^{2}$, of $\mathrm{CD}_{4}$ and is conveniently expressed in terms of the rotational parameter $\Omega_{R}$ or $T_{R}$ defined by

$$
\hbar \Omega_{R} \equiv k T_{R} \equiv \hbar^{2} / 2 I_{0} \text {. }
$$

Neglecting effects associated with permutation symmetry of the four deuterons in $\mathrm{CD}_{4}{ }^{3,4}$ the rotational correlation 
function may be shown to be well approximated by Eq. (A13) of Bloom et al. ${ }^{6}$ At high temperatures ( $T>T_{R}=3.7 \mathrm{~K}$ ) this equation yields the following expression for the reduced rotational correlation function:

$$
\begin{aligned}
{[g(t)]_{R}=} & 1 / 5 \int_{0}^{\infty} d J P_{J} \sum_{n=-2}^{+2} \\
& \times \cos \left\{2 n J[1+(n+1) / 2 J] \Omega_{R} t\right\},
\end{aligned}
$$

where, for a spherical top in the high-temperature approximation,

$$
\begin{aligned}
P_{J}= & (2 J+1)^{2} \exp \left[-J(J+1) T_{R} / T\right] / \int_{0}^{\infty}(2 J+1)^{2} \\
& \times \exp \left[-J(J+1) T_{R} / T\right] d J .
\end{aligned}
$$

The five values of the index $n$ arise from nonzero matrix elements of $Y_{2 m}[\Omega(t)]$ for $\Delta J=0(n=0), \Delta J= \pm 1$ $(n= \pm 1)$, and $\Delta J= \pm 2(n= \pm 2),{ }^{6}$ and their frequency factors $2 n J \Omega_{R}$ correspond to the (high-temperature approximation) $Q(\Delta J=0), \quad P, R \quad(\Delta J= \pm 1)$, and $O, S$ $(\Delta J= \pm 2$ ) branches of the rotational Raman spectrum of $\mathrm{CD}_{4}$ molecules. ${ }^{17}$ The integrals in Eq. (6) evaluated at high $J$ (i.e., $J>1$ ) give

$$
[g(t)]_{R}=1 / 5 \sum_{n=-2}^{+2}\left(1-n^{2} \omega_{R}^{2} t^{2}\right) \exp \left(-n^{2} \omega_{R}^{2} t^{2} / 2\right),
$$

where

$$
\omega_{R}=\left(k T / I_{0}\right)^{1 / 2}
$$

defines the free rotation parameter $\omega_{R}$. A plot of $[g(t)]_{R}$ vs $t$ is shown in Fig. 5. The areas above and below $[g(t)]_{R}=0.2$ indicated by cross hatching in this plot are equal and are associated with the oscillatory nature of the contributions of the $n= \pm 1, \pm 2$ terms to $[g(t)]_{R}$.

For a given $[g(t)]_{C}$ the relationship between $\tau_{\theta}$ and $\tau_{C}$ is given by Eqs. (2), (3), (4), and (8). For the limiting case $\omega_{R} \tau_{C}<1$ (strong friction limit) $\tau_{\theta}=\tau_{C}$ and for $\omega_{R} \tau_{C}>1$ (weak friction limit) $\tau_{\theta}=\tau_{C} / 5$, independent of the choice of $[g(t)]_{c}$. The passage from the strong to the weak friction limit $\left(\omega_{R} \tau_{C} \sim 1\right)$ is illustrated in Fig. 6 for $[g(t)]_{C}$ $=\exp \left(-t / \tau_{C}\right)$. As may be seen the variation of $\tau_{\theta}$ with $\tau_{C}$ is linear in the strong and weak friction limits but is a more slowly varying function of $\tau_{C}$ in the intermediate region because the area under the correlation function vs time curve is a slowly varying function of $\tau_{C}$ under those conditions.

In spectroscopic terms, the passage from the strong to the weak friction limit can be understood in terms of the widths of the rotational lines becoming smaller as $\tau_{c}$ increases. In the weak friction limit, the $J \neq 0$ rotational Raman lines contribute negligibly to the low-frequency spectral density.

Model B. Assumption that collisions change the angular momentum of the molecules and that the molecules rotate freely between collisions: This "extended diffusion model" was first developed for diatomic molecules by Gordon. ${ }^{18}$ The model assumes that the duration of a single collision is $<2 \pi / \omega_{R}$. In this model $\tau_{C}$ is the mean time between collisions which either randomize both the magnitude and the orientation of the angular momentum of the molecule ( $J$

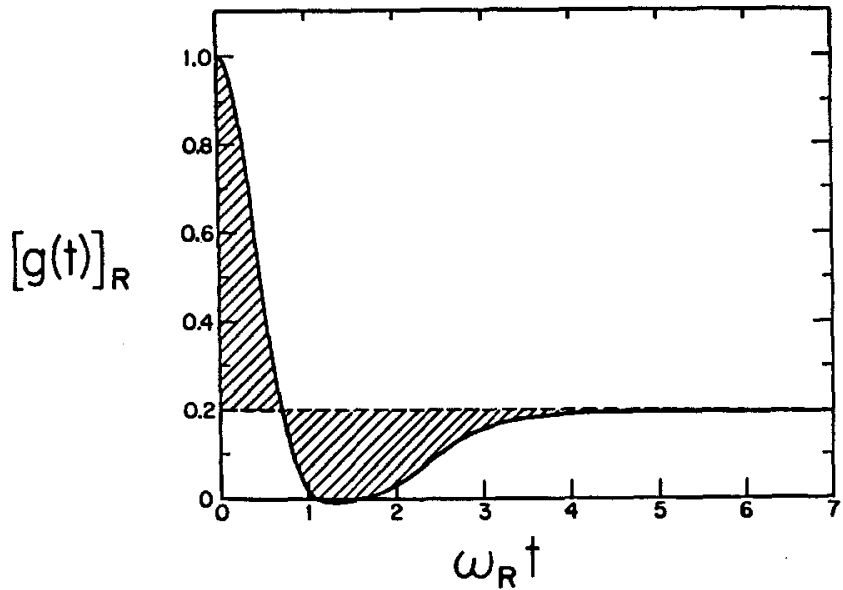

FIG. 5. The correlation function $[g(t)]_{R}$ vs $\omega_{R} t$ for freely rotating spherical top molecules in the classical limit. The shaded regions above and below $[g(t)]_{R}=0.2$ have equal areas.

diffusion) or only its orientation ( $M$ diffusion). Unlike model $\mathbf{A}$, the orientation of the molecule is not changed by the collision but evolves after the collision. The extended diffusion model has been applied to spherical top molecules by $\mathrm{McClung}^{19}$ who was written a comprehensive review ${ }^{20}$ of the application of the extended diffusion models to the interpretation of various spectroscopic measurements of molecular reorientation in fluids. The result of McClung's analysis for $J$ diffusion is that $\tau_{\theta}$ is given by

$$
\tau_{\theta}^{(2)} \equiv \tau_{\theta} \equiv j(0)=\tau_{C} X /(1-X),
$$

where

$$
\begin{aligned}
X= & 4 /\left(5 \pi^{1 / 2}\right) \sum_{n=-2}^{+2} \int_{0}^{\infty} d x x^{2} \exp \left(-x^{2}\right) / \\
& \left(1+2 n^{2} \omega_{R}^{2} \tau_{C}^{2} x^{2}\right) .
\end{aligned}
$$

Numerical calculations of Eq. (10) are plotted in Fig. 6 as $\omega_{R} \tau_{\theta}$ vs $\omega_{R} \tau_{C}$. As noted by McClung, ${ }^{19}$ this extended $J$ diffusion model gives the value $\tau_{\theta}=\tau_{C} / 4$ in the weak friction limit. A striking feature of this plot is that $\tau_{\theta}$ is predicted to go through a minimum corresponding to $\omega_{R} \tau_{\theta} \sim 0.65$ in

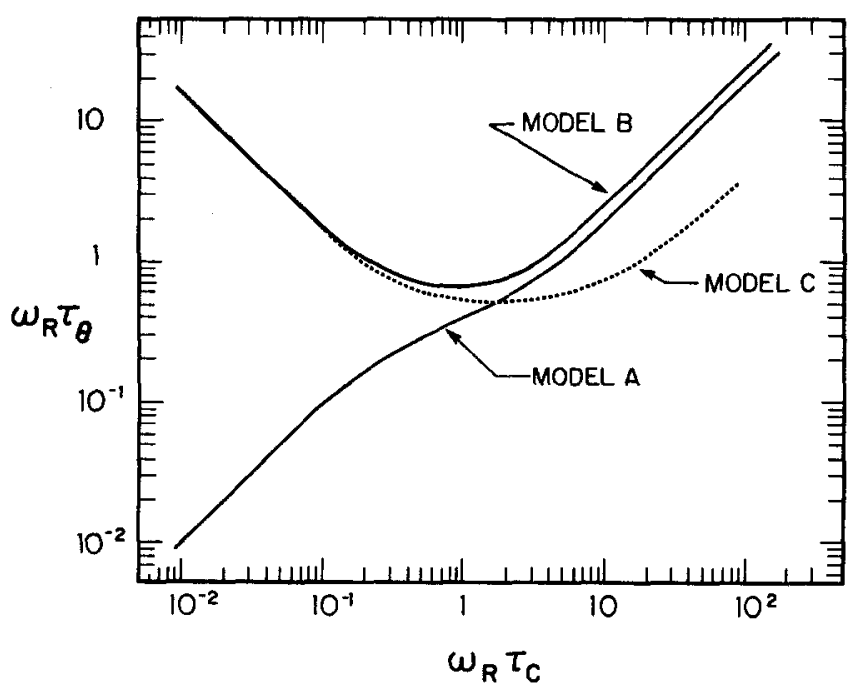

FIG. 6. $\omega_{R} \tau_{\theta}$ vs $\omega_{R} \tau_{C}$ for models A (strong collision), B (extended $J$ diffusion), and C (Fokker-Planck-Langevin). 
the vicinity of $\tau_{C} \sim \omega_{R}^{-1}$. This implies that as the molecular collision frequency is increased from low values (e.g., as in the dilute gas), the rate of molecular reorientation should increase, going through a maximum value where the collision frequency matches the average rotational frequency. This is a physically reasonable result. When $\omega_{R} \tau_{C}>1$, the randomization of the angular momentum by each collision and the subsequent free rotation of the molecule through large angles between successive collisions results in a randomization of the molecular orientation during the time $\tau_{C}$ between successive collisions. On the other hand, when $\omega_{R} \tau_{C}<1$, the molecules undergo only small rotations between successive collisions. Thus, the orientation of the molecule performs a random walk such that the mean squared rotation about a given axis in a time $t$ is proportional to the number of collisions $\left(t / \tau_{C}\right)$ times the mean squared rotation angle $\left(\omega_{R} \tau_{C}\right)^{2}$ between successive collisions, i.e., $\left\langle\theta^{2}\right\rangle_{t} \propto t / \tau_{C} \times\left(\omega_{R} \tau_{C}\right)^{2} \propto t \tau_{C}$. Since $t=\tau_{\theta}$ for $\left\langle\theta^{2}\right\rangle \sim 1$, $\tau_{\theta} \equiv \tau_{\theta}^{(2)} \propto \tau_{\mathrm{C}}^{-1}$ in this regime. Detailed calculations give the result $\tau_{\theta}^{(2)} \tau_{C}=I_{0} / 6 k T$ appropriate to the Debye limit. ${ }^{19,21}$

Model C. Assumption that molecules experience continuous intermolecular torques which produce only minute changes in angular velocity: An alternative and quite general treatment of rotational diffusion in dense fluids is provided by the Fokker-Planck-Lagevin FPL model ${ }^{22}$ which has been further developed and compared with the extended diffusion and other models in a number of papers. ${ }^{23-26}$ Molecules in the FPL model experience continuous intermolecular torques which produce only minute changes in the angular velocity. The molecule is assumed to be an appropriately shaped solid object immersed in a continuous viscous fluid in which the intermolecular torques are proportional to the angular velocity. The model is based on a rotational Fokker-Planck equation for the angular momentum-orientation conditional probability density and a rotational Langevin equation for the angular velocity. The FPL model has been quite successful in the joint analysis of data from Raman and NMR measurements of pure $\mathrm{CF}_{4}$ and of mixtures of $\mathrm{CF}_{4}$ in $\mathrm{Ar}$ and $\mathrm{Ne}$ at high pressures. ${ }^{25}$ In that work it was shown that the FPL model described the data much more accurately than the extended $J$-diffusion model (our model B).

Numerical calculations for the FPL model using the polynomial expansion of Eq. (4) and Table I of Ref. 24 for values of $\omega_{R} \tau_{C}<2$ and estimated from Fig. 8 of Ref. 24 and Fig. 1 of Ref. 25 for $\omega_{R} \tau_{C}>2$ are plotted in Fig. 6 as model C. As was the case for model $\mathrm{B}$, a minimum is again predicted. One way of distinguishing between the extended diffusion and FPL models is that $\omega_{R} \tau_{\theta}$ is $\sim 0.53$ at the minimum in the FPL model, approximately $18 \%$ less than for the extended $J$-diffusion model (see Fig. 6). Also, as seen in Fig. 6, the plot of $\omega_{R} \tau_{\theta}$ vs $\omega_{R} \tau_{C}$ exhibits a much broader minimum in the FPL than in the extended $J$-diffusion model.

\section{DISCUSSION}

In order to interpret the results of the $\mathrm{CD}_{4}$ relaxation measurements in liquid and solid solvents, it will be helpful to first examine the gas-phase measurements. On the basis of the gas-phase results, we shall show that the $\mathrm{CD}_{4}$ molecules reside in gas pockets for solvents in the solid state. By contrast, for each of the liquid solvents studied, $\tau_{\theta}$ of $\mathrm{CD}_{4}$ was found to be far too short to be explained in terms of gas pockets. In fact, we shall show that $\omega_{R} \tau_{C}$ is $\sim 1$ which represents the interesting regime between the strong and weak friction limits in which $j(0)=\tau_{\theta}$ is predicted to be relatively insensitive to $\tau_{C}$. We believe that this is the underlying reason for the relative insensitivity of the relaxation rate in liquid solvents to the temperature and the chemical composition of the solvent.

\section{Relaxation in $\mathrm{CD}_{4}$ gas}

The relaxation rate $R$ in $C_{4}$ gas can be expressed by Eqs. (1) and (2) so long as $\omega_{0} \tau_{\theta}<1$. The largest $R$ in the gas samples in Fig. 4 is $56 \mathrm{~s}^{-1}$ at 0.1 atm which yields $\tau_{\theta}=116$ ps and $\omega_{0} \tau_{\theta}=0.045$ at $\omega_{0} / 2 \pi=61.4 \mathrm{MHz}$. A maximum in $R$ will occur when $\omega_{0} \tau_{\theta} \sim 1$. The relaxation cross sections for $\mathrm{CH}_{4}$ and $\mathrm{CD}_{4}$ are roughly equal. ${ }^{6}$ Therefore the values of $\tau_{\theta}$ for these two molecules will be similar, and the maxima in the gas-phase values of $R$ will occur at roughly the same pressure. For room temperature $\mathrm{CH}_{4}$ gas at $30 \mathrm{MHz}$ this pressure is $0.04 \mathrm{~atm}^{3,4}$; the pressure at $61.4 \mathrm{MHz}$ will be approximately $0.02 \mathrm{~atm}$. Except for the $0.1 \mathrm{~atm}$ sample, our pressures are well above these values so that the short correlation time approximation is well satisfied. Since the gas pressures are sufficiently low that the weak friction limit also holds, the results of the theoretical section predict that $R \propto \tau_{\theta} \propto \tau_{C} ; \tau_{C}$, the mean time between collisions, is $\propto P^{-1}$, where $P$ is the pressure. We find that over the range of pressures studied at $300 \mathrm{~K}$, the plot of $R^{-1}$ vs $P$ is indeed linear but that the line does not pass through the origin. Our empirical fit to the experimental results in Fig. 4 is

$$
R^{-1}=C\left(P+P_{0}\right),
$$

where $P_{0}=0.23 \mathrm{~atm}$ and $C=0.0835 \mathrm{~s}^{-1} \mathrm{~atm}^{-1}$. The reason that the fit of Eq. (12) does not extrapolate to the origin is that the quadrupolar interaction is modulated by the centrifugal distortion (rotation-vibration) interaction which partially removes the $(2 J+1)^{2}$ degeneracy of each set of $J$ states. ${ }^{3,4}$ Many of the populated rotational states at $300 \mathrm{~K}$ have centrifugal distortion splittings of the same order of magnitude as the collision induced reorientation frequency over the range of pressures studied in our experiments. Similar effects have been explored experimentally and theoretically in studies of proton Zeeman relaxation in $\mathrm{CH}_{4}$ gas. ${ }^{3,4}$ The main difference between the $\mathrm{CH}_{4}$ and $\mathrm{CD}_{4}$ systems is that the relaxation in $\mathrm{CH}_{4}$ is due to spin-rotation interactions while that in $\mathrm{CD}_{4}$ is due to quadrupolar interactions. Examination of Figs. 3 and 4 in Ref. 3 shows that the plot of $R^{-1}$ vs $P$ in $\mathrm{CH}_{4}$ is similar to our observations over the pressure range of 1 to $10 \mathrm{~atm}$. Note that the difference between curves (B) and (D) of Fig. 4 of Ref. 3 is due to the centrifugal distortion terms. A detailed investigation of nuclear spin relaxation in $\mathrm{CD}_{4}$ gas would involve a study over a wide range of densities and Larmor frequencies.

\section{Relaxation of $\mathrm{CD}_{4}$ "dissolved" in sollds}

A true homogeneous solid solution would be expected to yield $\mathrm{CD}_{4}$ relaxation rates that are similar to those found 
in liquid solutions of equal pressure. Our results do not agree with this picture because the observed $R$ values (Fig. 3) are far too large. However, if we assume that the solute $C_{4}$ molecules reside in gas pockets in the solid, we can use our empirical fit to the gas-phase results [Eq. (12)] to compute $R$ from the known gas densities. For all samples and all $C_{4}$ pressures ( 1 to $18 \mathrm{~atm}$ ) used, the resulting values, corrected for temperature using the ideal gas law, are within $25 \%$ of the values measured when the solvent is in the solid state. This is strong evidence that the $\mathrm{CD}_{4}$ molecules do indeed exist in gas pockets. It is not possible to say anything precise about the size of these gas pockets on the basis of the current measurements since we have no information on the effects of wall collisions on the molecular orientation. However, the size of the pockets could be determined by spin-echo studies of bounded translational diffusion. ${ }^{27}$

\section{Relaxation of $\mathrm{CD}_{\mathbf{4}}$ dissolved in liquids}

When the preceding analysis for gas pockets is applied to the $R$ vs $T$ results for the liquid state of the samples it implies pressures of hundreds of atmospheres of $\mathrm{CD}_{4}$. This is orders of magnitude greater than the known pressure and implies that the $\mathrm{CD}_{4}$ is not in gas pockets when the solvent is in the liquid state. Indeed this is consistent with the observation that both the 1 and $15 \mathrm{~atm}$ samples of $\mathrm{CD}_{4}$ in benzene give essentially the same $R$ vs $T$ curve when the solvent is in the liquid state. This also suggests that whatever the unknown concentrations of $\mathrm{CD}_{4}$ in the liquid state of the solvent, we can safely assume an infinite dilution limit.

The results of Fig. 2 indicate that $R$ is a relatively insensitive function of temperature, pressure, and solvent. If such results were interpreted according to model $A$, they would imply a smaller variation of $\tau_{C}$ than can reasonably be expected for such a wide variation of physical conditions. By contrast, models B and C offer a reasonable explanation for the insensitivity of $R$ providing that the experimental values of $\tau_{\theta}$ are close to the $\omega_{R} \tau_{\theta}$ minimum, which corresponds to $\left(\omega_{R} \tau_{\theta}\right)_{\min } \sim 0.65$ for model B and $\sim 0.53$ for model C. Examination of Table I shows, indeed, that the range of values obtained for $\omega_{R} \tau_{\theta}$ from the measurement of $R$ in each of the solvents has a lower bound above and in some cases equal to $\left(\omega_{R} \tau_{\theta}\right)_{\min }=0.65$, within the experimental error of about $10 \%$. It is interesting that, in some of the plots of $R$ vs temperature in Fig. 2 [see especially Figs. 2(a), 2(b), and 2 (e) ], there are clear indications that $R$ goes through a minimum as the temperature is changed. According to McClung's theory of the extended $J$-diffusion model (see Fig. 1 of Ref. 19 and Fig. 6 of this paper), the collision frequency at $\left(\omega_{R} \tau_{\theta}\right)_{\min }=0.65$ corresponds to $\tau_{C}^{-1}=\omega_{R}$. For the extended $M$-diffusion model (Fig. 2 of Ref. 19), the value of $\left(\omega_{R} \tau_{\theta}\right)_{\min } \sim 1$. Therefore, our measurements are in agreement with the predictions of the $J$-diffusion model but not with those of the $M$-diffusion model.

As discussed in the theoretical section, the FPL model (model C) also predicts a minimum value for $\omega_{R} \tau_{\theta}$; in this case the minimum is broad and value is predicted to be about $18 \%$ lower than that for the extended $J$-diffusion model. As described in Table I, our present knowledge of the quadrupole coupling does not allow us to distinguish between the
FPL and the extended $J$-diffusion models. If the value of $\left(e V_{z z} Q / h\right)$ in Eq. (1) turns out to be close to the recently proposed value of $\left(e V_{z z} Q / h\right)^{e q}=189 \mathrm{kHz}^{2,9}$ it would strongly favor the FPL model.

It is interesting that the values of $R \sim 0.1 \mathrm{~s}^{-1}$ obtained in liquid $\mathrm{CD}_{4}$ at temperatures close to $T=100 \mathrm{~K}^{28}$ also yield a value of $\omega_{R} \tau_{\theta} \sim 0.67$ very close to $\left(\omega_{R} \tau_{\theta}\right)_{\min }$.

\section{CONCLUDING REMARKS}

On the basis of a study of the spin-lattice relaxation rates of the deuteron spins in $\mathrm{CD}_{4}$ dissolved in the liquid and solid phases of several organic solvents, we conclude that the $\mathrm{CD}_{4}$ molecules reside in gas pockets in the solids and are uniformly dispersed in the liquids. A quantitative analysis of our relaxation measurements provides a striking demonstration of the validity of either the extended $J$-diffusion model or the Fokker-Planck-Langevin model for molecular reorientation of $\mathrm{CD}_{4}$ molecules dissolved in liquids. Both these models predict a minimum in the plot of relaxation rate vs collision frequency, i.e., a maximum in $T_{1}$. Our measurements support this prediction and, within the experimental uncertainty of about $10 \%$, yield the predicted value for $R$ at the minimum. A better determination of the vibrationally averaged quadrupolar coupling constant for the deuteron in $\mathrm{CD}_{4}$ will allow one to distinguish between the FPL and extended $J$-diffusion models using the experiments reported here. The results are not consistent with the extended $M$-diffusion model or with a strong collision model.

\section{ACKNOWLEDGMENTS}

We wish to thank The Natural Science and Engineering Research Council of Canada for financial support, Professor C. A. de Lange, Professor R.E.D. McClung, and Dr. J. G. Snijders for fruitful discussions, and the Ivanhoe for providing ambiance suitable for scientific discourse.

${ }^{1}$ M. Bloom and J. A. Morrison, in Surface and Defect Properties of Solids, edited by M. W. Roberts and J. M. Thomas (The Chemical Society, London, 1973), Vol. 2, pp. 140-159.

${ }^{2}$ J. G. Snijders, C. A. de Lange, and E. E. Burnell, Isr. J. Chem. 23, 269 (1983).

${ }^{3}$ P. A. Beckmann, M. Bloom, and I. Ozier, Can. J. Phys. 54, 1712 (1976). ${ }^{4}$ P. A. Beckmann, M. Bloom, and E. E. Burnell, Can. J. Phys. 50, 251 (1972).

${ }^{5} \mathrm{M}$. Bloom, MTP International Review of Science, Physical Chemistry, Series One, edited by C. A. McDowell (Butterworths, London, 1972), Vol. 4, pp. $1-42$.

${ }^{6}$ M. Bloom, F. Bridges, and W. N. Hardy, Can. J. Phys. 45, 3533 (1967).

${ }^{7}$ E. E. Burnell and C. A. de Lange, J. Chem. Phys. 76, 3474 (1982).

${ }^{8}$ J. G. Snijders, C. A. de Lange, and E. E. Burnell, J. Chem. Phys. 77, 5386 (1982).

${ }^{9}$ J. G. Snijders, C. A. de Lange, and E. E. Burnell, J. Chem. Phys. 79, 2964 (1983).

${ }^{10}$ R. Ader and A. Loewenstein, Mol. Phys. 24, 455 (1972).

${ }^{11}$ R. Ader and A. Loewenstein, Mol. Phys. 30, 199 (1975).

${ }^{12}$ A. Loewenstein, Chem. Phys. Lett. 38, 543 (1976). 
${ }^{13}$ A. Abragam, The Principles of Nuclear Magnetism (Oxford University, Oxford, 1961).

${ }^{14}$ L. J. Burnett and B.H. Muller, J. Chem. Phys. 55, 5829 (1971).

${ }^{15}$ M. F. Brown, J. Seelig, and U. Häberlen, J. Chem. Phys. 70, 5045 (1979).

${ }^{16}$ P. A. Beckmann, J. W. Emsley, G. R. Luckhurst, and D. L. Turner, Mol. Phys. 50, 699 (1983).

${ }^{17}$ R. G. Gordon, J. Chem. Phys. 45, 1649 (1966).

${ }^{18}$ R. G. Gordon, J. Chem. Phys. 44, 1830 (1966).

${ }^{19}$ R. E. D. McClung, J. Chem. Phys. 51, 3842 (1969).

${ }^{20}$ R. E. D. McClung, Adv. Mol. Relaxation Interaction Processes 10, 83 (1977).
${ }^{21}$ P. S. Hubbard, Phys. Rev. 131, 1155 (1963).

${ }^{22}$ M. Fixman and K. Rider, J. Chem. Phys. 51, 2425 (1969).

${ }^{23}$ R. E. D. McClung, J. Chem. Phys. 73, 2435 (1980).

${ }^{24}$ G. Lévi, J. P. Marsault, F. Marsault-Hérail, and R. E. D. McClung, J. Chem. Phys. 73, 2443 (1980).

${ }^{25}$ S. Perry, V. H. Schiemann, M. Wolfe, and J. Jonas, J. Chem. Phys. 85, 2805 (1981).

${ }^{26}$ J. G. Powles and G. Rickayzen, Mol. Phys. 33, 1207 (1977).

${ }^{27}$ R. C. Wayne and R. M. Cotts, Phys. Rev. 151, 264 (1966).

${ }^{28}$ G. A. de Wit and M. Bloom, Can. J. Phys. 43, 986 (1965). 